\title{
Determination of the full-genome sequence of hepatitis E virus (HEV) SAAS-FX17 and use as a reference to identify putative HEV genotype 4 virulence determinants
}

\author{
Yumin Zhu ${ }^{1,2}$, Xiaoming $\mathrm{Yu}^{3}$, Fenfen Huang ${ }^{4}$, Ruisong $\mathrm{Yu}^{1,2}$, Shijuan Dong ${ }^{1,2}$, Fusheng $\mathrm{Si}^{1,2}$, Yuanshu Zhang ${ }^{3}$ \\ and Zhen $\mathrm{Li}^{1,2^{*}}$
}

\begin{abstract}
Background: Four major genotypes of hepatitis E virus (HEV), the causative agent of hepatitis E, have so far been recognized. While genotypes 3 and 4 are both zoonotic, the disease symptoms caused by the latter tend to be more severe. To examine if specific nucleotide/amino acid variations between genotypes 3 and 4 play a role in determining the severity of hepatitis $E$ disease, the complete genome of one swine HEV genotype 4 isolate, SAAS-FX17, was determined and compared with other genotype 4 and genotype 3 genomes to identify putative HEV genotype 4 virulence determinants.

Results: A total of 42 conformable $\mathrm{nt} / \mathrm{aa}$ variations between genotype 3 and $4 \mathrm{HEV}$ s were detected, of which 19 were proposed to be potential disease severity determinants for genotype 4 strains.

Conclusions: One potential determinant was located in each of the 5'-UTR and 3'-UTR, 3 and 12 within ORF1 and ORF2 respectively, and 2 in the junction region.
\end{abstract}

Keywords: Hepatitis E virus, Disease severity determinant, Subtype, Genotype, Zoonosis

\section{Background}

Hepatitis E virus (HEV) is an important human pathogen in many regions of the world, and is the causative agent of acute hepatitis, a disease spread mainly through fecal contamination of water supplies or food [1]. HEV is the sole member of the genus Hepevirus of the family Hepeviridae [2]. The virus is a single-stranded positivesense RNA virus containing a short $5^{\prime}$ untranslated region (UTR), three open reading frames (ORF1-3), and a 3' UTR [3]. ORF1 encodes non-structural proteins involved in viral replication, ORF2 encodes a structural protein comprising the virion capsid, and ORF3, which overlaps ORF2, is required for viral egress from infected cells [4].

\footnotetext{
*Correspondence: zhenli60@163.com

'Institute of Animal Science and Veterinary Medicine, Shanghai Academy of Agricultural Sciences, Shanghai 201106, China

${ }^{2}$ Shanghai Key Laboratory of Agricultural Genetics and Breeding, Shanghai 201106, China

Full list of author information is available at the end of the article
}

Four major HEV genotypes and several subtypes within each genotype have been identified in mammalian species [5]. Genotypes 1 and 2 have been isolated from humans only and genotypes 3 and 4 are zoonotic [6]. Evidence is accumulating to indicate that different HEV genotypes are associated with disease symptoms of differing severity. For example, genotypes 3 and 4 appear to be less virulent for humans compared with genotypes 1 and 2 [7]. Furthermore, disease symptoms caused by genotype 4 are reported to be more severe compared with genotype 3 infections [8-10], and genotype 4 viral loads recorded in a co-infected patient were also higher [11].

In order to identify specific nucleotides/amino acids influencing the severity of HEV infections, we have now determined the full genome sequence of swine HEV strain, SAAS-FX17, which was previously classified as a genotype 4, sub-type (i) virus [12]. Potential determinants responsible for the observed variations in the 
severity of disease symptoms caused by genotype 3 and 4 strains were then identified by comparison with the corresponding sequences of 56 genotype 4 and 56 genotype 3 HEV's retrieved from GenBank.

\section{Materials and methods}

Fecal specimens and extraction of RNA

Swine HEV, strain SAAS-FX17, was isolated from a fecal specimen collected in 2008 from a pig farm located in a Shanghai suburb. Sample treatment and total RNA extraction procedures were described previously [13].

\section{Reverse transcription and PCR}

All the non-terminal reverse transcripts (RTs) were synthesized with the SuperScript ${ }^{\mathrm{TM}}$ III First-strand Synthesis System (Invitrogen, USA) using an external antisense primer, and the first-strand cDNA was used immediately for PCR.

Ten sets of specific external and internal primer pairs (see Additional file 1: Table S1) were used to amplify the entire viral genome. Nucleotide (nt) sequences at the $5^{\prime}$ and 3 ' ends were determined with the SMART ${ }^{\mathrm{TM}}$ Rapid Amplification of cDNA Ends (RACE) cDNA Amplification Kit (Clontech Laboratories, Japan) according to the manufacturer's instructions.

\section{Sequence analysis}

PCR products were purified and ligated into a pJET 1.2/ blunt cloning vector (Fermentas). At least three positive clones were selected at random and sequenced (Sangon Biotech Shanghai Co., Ltd) in both directions using an ABI model 3730 automatic DNA sequencer (ABI, CA, USA).

Sequence assembly was accomplished and percent identity values were calculated using Lasergene (version 7.10; DNAstar). Sequence alignments were generated by Clustal-W (version 1.8). Phylogenetic trees were constructed by the neighbor-joining method with the aid of the MEGA 4.0 software package. Genetic distances were calculated by using the Kimura two-parameter method.

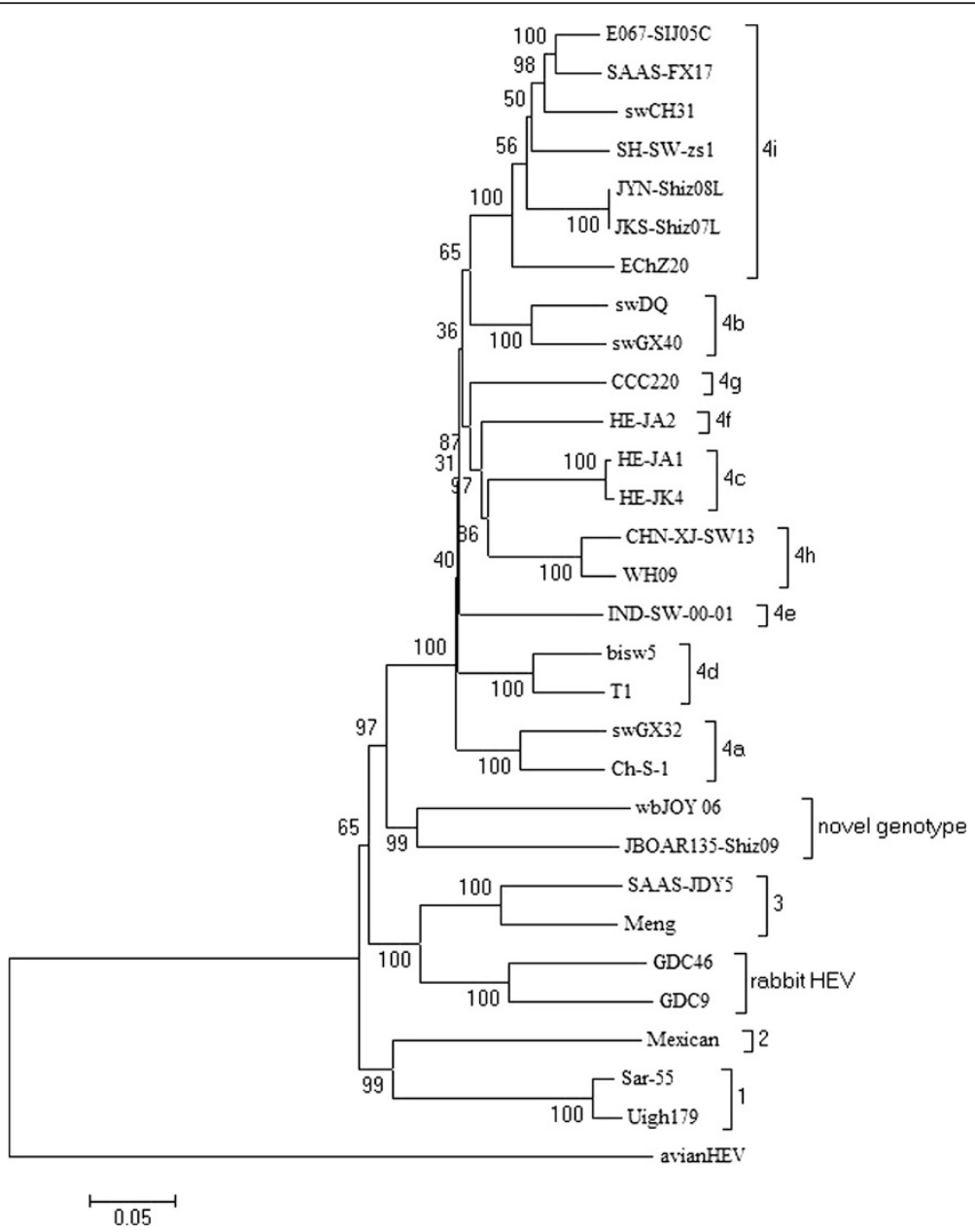

Figure 1 Phylogenetic tree depicting the genotypic/subgenotypic status of $30 \mathrm{HEV}$ isolates based on the full length genomic sequence. Internal node numbers indicate the bootstrap values as a percentage of trees obtained from 1000 replicates. 
One thousand re-samplings of the data were used to calculate branch percentage values. Secondary structures in the 5' UTR were predicted with the mfold program [14].

\section{Nucleotide sequence accession number}

The full genomic nucleotide sequence of strain SAASFX17 has been deposited in the GenBank database under accession number JF915746. Accession numbers and classifications of the $120 \mathrm{HEV}$ reference strains are shown in Additional file 2: Table S2. Of these, 30 complete HEV genome sequences were selected at random in order to construct the genotype/subtype phylogenetic tree (Figure 1). All nt and amino acid (aa) comparisons are made with reference to strain SAAS-FX17.

\section{Results}

Genome organization of swine HEV strain SAAS-FX17

The full-length genome of strain SAAS-FX17 consisted of $7262 \mathrm{nt}$, excluding the poly (A) tail at the 3 ' terminus, and contained three ORFs. The genome was organized into a 5' UTR extending from nt 1-26, ORF1 from nt 27-5135 (5109 nt), ORF2 from 5174-7156 (1983 nt), and a smaller ORF3 (which overlapped ORF2) from 5166-5504 (339 nt). The 77 nt $3^{\prime}$ UTR extended from 7157 to 7233 and was followed by a poly (A) tail of 29 A residues.

\section{Phylogenetic analysis}

The nt sequence of SAAS-FX17 was 74.5-75.7\% similar to all recorded HEV genotypes $1-3$ and was $83.7-94.8 \%$ identical with the genome sequences of all reported genotype 4 HEVs. Consistent with an earlier classification [12], SAAS-FX17 was confirmed as belonging to subtype $4 \mathrm{i}$ within genotype 4 (Figure 1 ). Sequence similarity with other viruses within this subgroup was 90.6-94.8\%, and $84.0-85.6 \%$ with members of the other genotype 4 sub-groupings (4a-4h).

The SAAS-FX17 genome is most closely related to that of a human HEV strain, E067-SIJ05C, derived from an acute hepatitis $\mathrm{E}$ patient in Japan, who had traveled to Shanghai prior to the onset of disease symptoms. These two isolates shared nt similarities of 94.8\%, 94.6\%, $95.1 \%$ and $97.6 \%$ in terms of the full-length genome, ORF1, ORF2 and ORF3, respectively.

\section{5' UTR and 3' UTR analyses}

The 5' UTR of SAAS-FX17 consisted of 26 nt and contained one additional $\mathrm{G}$ at the extreme 5 ' terminus compared with the majority of genotype 4 strains. One unique nt substitution (C23T) was identified in the second loop structure of the 5' UTR of genotype $4 \mathrm{HEV}$ strains which, as seen in Figure 2, contained eight nts compared with six for genotype 3 strains.
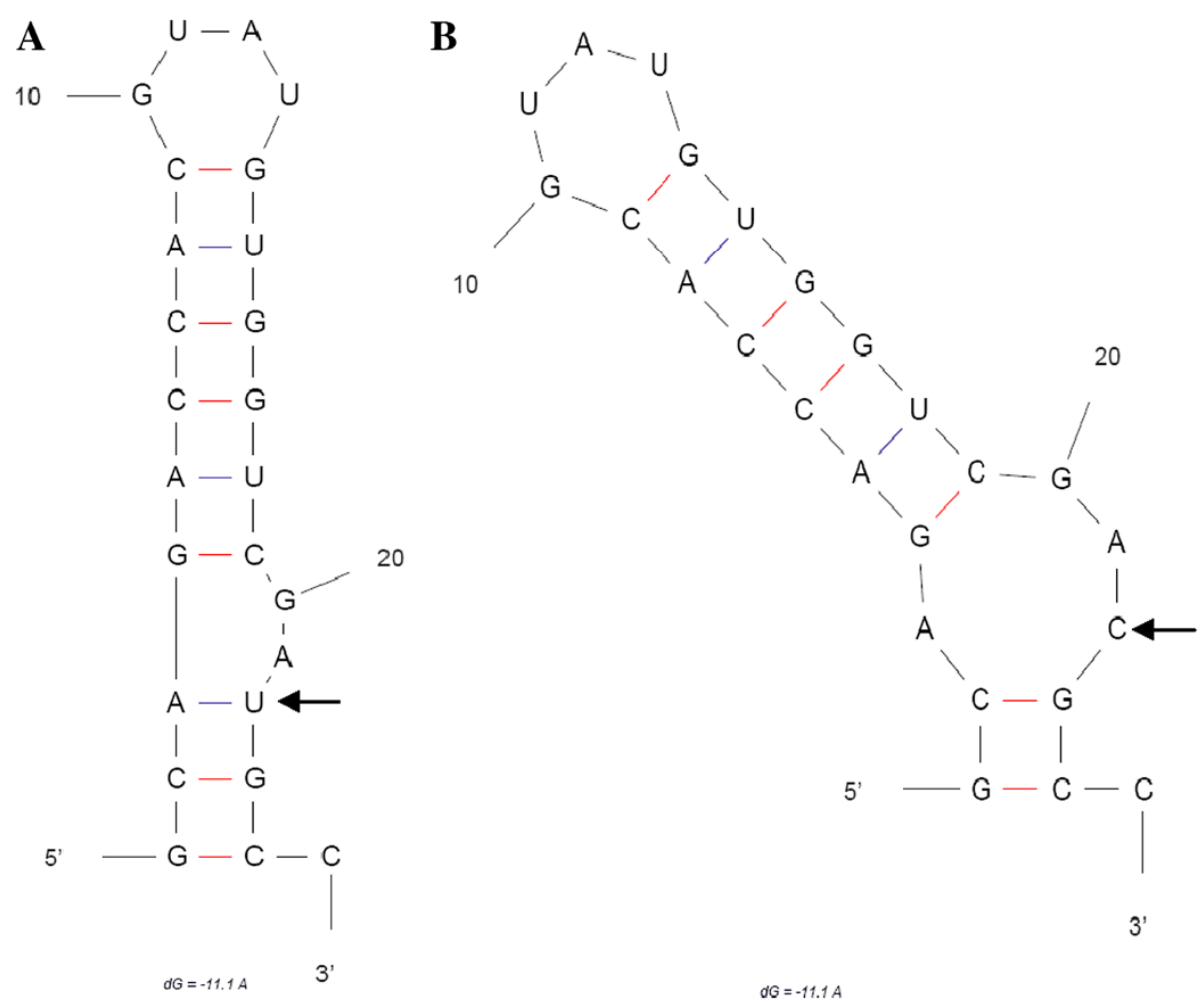

Figure 2 Secondary structures of the 5'-UTR region of HEV genotypes 3 (A) and genotypes 4 (B), as predicted by the mfold program. Arrows indicates the nucleotide variation between the different genotypes. 
SAAS-FX17 contained a 3' UTR consisting of $77 \mathrm{nt}$ excluding the poly (A) tail but including five additional nts (ACTGGT) at the terminus. 3' UTRs of all genotype 4 strains contained an initial TTTATT sequence as opposed to TTAATT in the 3' UTRs of genotype 3 strains.

\section{Analysis of ORFs \\ ORF1}

The ORF1 of SAAS-FX17 consisted of 5109 (27-5135) nts capable of encoding a protein of 1702 aa. The nucleotide and amino acid sequences were $82.5-94.6 \%$ and 93.1-98.2\% identical, respectively to the corresponding sequences of other genotype 4 isolates.

Excluding the hypervariable region (HVR), 12 genotype 4 specific aa substitutions were identified in the ORF1 (Table 1). Two aa variations were evident in the HVR, the initial conserved sequence of which was VSGFSSCFSP in genotype 4 strains compared with TSGFSSDFSP in genotype 3 strains (Table 1).

\section{ORF2}

The ORF2 of SAAS-FX17 consisted of 1983 nts capable of encoding a protein of 660 aa. The nucleotide and amino acid sequences were $86.7-95.1 \%$ and $93.9-99.1 \%$ identical, respectively to the corresponding sequences of other genotype 4 isolates. Comparison of the ORF2 proteins in the two HEV genotypes revealed a total of 18 aa substitutions (Table 1).

\section{ORF3}

The ORF3 of SAAS-FX17 consisted of 345 nts, encoding 114 aa. The nucleotide and amino acid sequences were 91.3-98.8\% and $90.3-97.7 \%$ identical, respectively to the corresponding sequences of other genotype 4 isolates. The ORF3-encoded protein of genotype 4 strains contained two aa insertions (5P, 84Q) and one aa deletion (aa residue 68), an additional aa overall compared with genotype 3 viruses. Furthermore, there was an a substitution (A35V) when compared with genotype 3 (Table 1).

\begin{tabular}{|c|c|}
\hline Region & Amino acid variation and site \\
\hline ORF1 & $\begin{array}{l}\text { Substitutions: Q486E, R491L, E494D, A501E, F502P/L/E, E503ANN } \\
\text { S505L/N/I/F, D508S, P509G, A510T/S, T/A524H/Y/T, D1574N }\end{array}$ \\
\hline $\mathrm{HVR}$ & Substitutions: T707V, D713C \\
\hline ORF2 & $\begin{array}{l}\text { Substitutions: 166V, P/S67V/F/L, T/A68S/P, A/T69Q, T/A70S/P, } \\
\text { P/S97A/TN, A114S, T/A146S, I147V, T149S/A, S161N, S324T, } \\
\text { S/G326T, K330R, G509A, C580A, N587S, S590A }\end{array}$ \\
\hline ORF3 & $\begin{array}{l}2 \text { aa insertions (5P, 84Q) , } 1 \text { aa substitution (A35V) and } 1 \text { aa } \\
\text { deletion (aa residue 68) }\end{array}$ \\
\hline
\end{tabular}

\section{Junction region (JR)}

The JR[15] of SAAS-FX17 consisted of 38 bp, which is identical in length to most other genotype 4 strains but 4 bp longer than the corresponding region of genotype 3 strains. These additional $4 \mathrm{bp}$ were due to $4 \mathrm{nt}$ insertions at sites 10, 30, 31 and 32 of the JR. The space length (distance between the ORF2 and ORF3 initiation sites) was $11 \mathrm{bp}$ and $8 \mathrm{bp}$ for genotypes 4 and 3, respectively (Figure 3).

\section{Discussion}

Since 2000, genotype $4 \mathrm{HEV}$ has replaced genotype 1 as the dominant cause of hepatitis $\mathrm{E}$ in China [16-18]. Recently, Zhu et al. [12] identified a novel virus belonging to subtype $4 \mathrm{i}$, the same subtype to which SAAS-FX17 has been assigned. In view of the close relationship between SAAS-FX17 and strain E067-SIJ05C, derived from an acute hepatitis $\mathrm{E}$ patient in Japan who had traveled to Shanghai before the onset of disease symptoms, it appears HEV strains belonging to this subtype may undergo zoonotic transmission.

Zhang et al. [19] previously suggested that the 5' UTR of the viral genome may play an important role in replication and/or translation. Other researchers reported that the $5^{\prime}$ UTR and a conserved 58 nt region within ORF1 were likely to fold into conserved stem-loop and hairpin structures, which were postulated to be important for HEV RNA replication [20]. In the present study, a unique nt substitution, C23T, was identified within the putative stem-loop structure of the $5^{\prime}$ UTR, which resulted in a change in the size of the second loop. Previous studies have shown that nt variations in the central portion of the 5' UTR may influence the severity of type A hepatitis [21]. However, although a potential virulence determinant for genotype $4 \mathrm{HEV}$, the impact of $\mathrm{C} 23 \mathrm{~T}$ on HEV disease symptoms remains to be established.

The 3' UTR and an adjacent region of the HEV genome form a putative stem-loop structure that affects the binding of recombinant viral RNA dependent RNA polymerase (RdRp) and initiation of RNA synthesis [22]. Graff et al. [23] showed that a seemingly minor change caused by a mutation at nt 7106 , which eliminated one putative base pair within the stem-loop, significantly inhibited RNA replication, and the magnitude of virus replication could be the reason for the severity difference of HEV. Therefore, based on these previous research findings, a single nt difference (T3A) identified between the 3' UTR sequences of HEV genotypes 3 and 4 also represents a putative virulence determinant.

HEV ORF1 encodes a large nonstructural protein with several putative functional motifs [24]. Our data derived from sequence comparisons of this region in HEV genotypes 3 and 4 revealed 12 specific aa substitutions, 11 of which occurred in the protease motif. However, since no 
functional activity relating to disease severity has so far been attributed to this enzyme, these substitutions are unlikely to have a role as virulence determinants. The remaining substitution was located in the RdRp motif, which is essential for genomic RNA replication. In addition, two aa variations were recorded in the conserved initiation motifs of the HVRs of genotype 3 and 4 strains. Virus attenuation that accompanied complete deletion of this region of ORF1 led to the suggestion that the HVR played a biological role in HEV pathogenesis [25]. Therefore, we propose that these two variations together with the substitution in the RdRp motif are potential candidates for virulence variation in type $4 \mathrm{HEV}$.

ORF2 encodes the viral capsid protein, including a signal peptide (aa 1-22) involved in the translocation of the protein from the endoplasmic reticulum [26], and an arginine-rich domain (aa 23-111) that may be involved in RNA encapsidation [27]. Córdoba et al. [28] recently verified that mutations within the latter domain contributed to virus attenuation. Our data comparing HEV genotypes 3 and 4 identified six specific aa substitutions within this region, five of which (aa residues 66-70) were continuous. This entire sequence motif may represent a single putative virulence determinant [16], implying the possible existence of two such determinants in the entire arginine-rich region. Three structural domains have been defined within the C-terminus of the HEV capsid: S (residues 118-313), P1 (residues 314-453) and P2 (residues 454-606), which function in forming the capsid shell, binding of the virus to host cell receptors, and antigenicity, respectively [29,30]. Single or multiple variations in the aa sequences of the capsid or envelope proteins resulted in attenuated viral phenotypes [31,32] and, more recently, Córdoba et al. [28] verified that $\mathrm{HEV}$ attenuation was also linked to mutations in the P1 domain. Our study revealed four, three and four specific substitutions in the S, P1 and P2 domains, respectively of genotype $4 \mathrm{HEV}$. Each might constitute individual putative virulence determinants 
although the proximate substitutions at positions 146147 could represent a single influencing factor.

ORF3 protein is essential for virion release from HEV infected cells [33]. However, it remains unclear if differences in the length of the ORF3 regions of HEV genotypes 3 and 4, or specific aa variations in the encoded proteins, influence the severity of the respective clinical symptoms.

The Junction Region (JR) denotes the genome segment between the stop codon of ORF1 and the putative initiation codon of ORF2, in which a bicistronic subgenomic mRNA encodes both ORF2 and ORF3 proteins of HEV [15]. Cao et al. [34] demonstrated that nt mutations or a mutation in the stem-loop structure formed within the JR significantly inhibited HEV replication. Furthermore, Shukla et al. [35] reported that the distance between the initiation codons of ORF2 and ORF3 affected initiation preferences. Therefore, nt mutations and distance variation between the ORF2 and ORF3 initiation codons within the JR of genotypes 3 and 4 may constitute strong candidates for determinants of disease severity. Although four nt insertions at sites 10, 30, 31 and 32 of the JR were identified in this study, the contiguous insertions at positions 30-32 may represent a single putative virulence determinant.

\section{Conclusions}

A total of $42 \mathrm{nt} / \mathrm{aa}$ variations between HEV genotypes 3 and 4 were identified. Not all may constitute potential virulence determinants since several observed contiguous nt/aa variations, which are often responsible for a single mutation incident [16,36], may each constitute a single determinant. Based on this conjecture, the $42 \mathrm{nt} / \mathrm{aa}$ variations represented 19 potential determinants. However, the possibility of additional putative determinants existing among the 42 variations cannot be excluded.

\section{Additional files}

Additional file 1: Table S1. Genomic position and nucleotide sequence of oligonucleotide primers for PCR. The genomic position refers to strain SAAS-FX17 (JF915746) adopted in this study. ES, EA, IS and IA denote 'external sense', 'external antisense', 'internal sense' and 'internal antisense', respectively.

Additional file 2: Table S2. Genotype, strain designation and GenBank accession numbers of $120 \mathrm{HEV}$ reference strains employed in this study.

\section{Competing interests}

The authors declare that there are no competing interests.

\section{Authors' contributions}

YZ carried out the full-genome sequence determination. YZ and ZL participated in the sequence alignment and drafted the manuscript. XY, FH, $\mathrm{RY}, \mathrm{SD}, \mathrm{FS}$ and YZ participated in parts of the study. All authors read and approved the final manuscript.

\section{Acknowledgments}

We thank Dr John Buswell, Institute of Edible Fungi, for linguistic revision of the manuscript. This work was supported by a grant from the Shanghai Municipal Natural Science Foundation (NO.09ZR1428100), and the Youth Scientists Program of the Shanghai Academy of Agricultural Sciences (SAAS) (2009(16)).

\section{Author details}

'Institute of Animal Science and Veterinary Medicine, Shanghai Academy of Agricultural Sciences, Shanghai 201106, China. ${ }^{2}$ Shanghai Key Laboratory of Agricultural Genetics and Breeding, Shanghai 201106, China. ${ }^{3}$ Veterinary College, Nanjing Agricultural University, Nanjing, Jiangsu 210095, China. ${ }^{4}$ College of Animal Science and Technology, Jiangxi Agricultural University, Nanchang, Jiangxi 330000, China.

Received: 15 June 2012 Accepted: 30 October 2012

Published: 8 November 2012

\section{References}

1. Meng XJ, Shivaprasad HL, Payne C: Hepatitis E virus infections. In Diseases of Poultry. 12th edition. Edited by Saif M, et al:: Blackwell Publishing Press; 2008:443-452.

2. Emerson SU, Anderson D, Arankalle A, Meng XJ, Purdy M, Schlauder GG, Tsarev SA, Fauquet CM, Mayo MA, Maniloff J, Desselberger U, Ball LA: Hepevirus. In Virus taxonomy. VIllth edition. London, United Kingdom: Elsevier/Academic Press; 2004:851-855.

3. Harrison TJ: Hepatitis E virus - an update. Liver 1999, 19:171-176.

4. Yamada K, Takahashi M, Hoshino Y, Takahashi H, Ichiyama K, Nagashima S, Tanaka T, Okamoto H: ORF3 protein of hepatitis E virus is essential for virion release from infected cells. J Gen Virol 2009, 90:1880-1891.

5. Lu L, Li C, Hagedorn CH: Phylogenetic analysis of global hepatitis E virus sequences: genetic diversity, subtypes and zoonosis. Rev Med Virol 2006, 16:5-36.

6. Meng XJ, Purcell RH, Halbur PG, Lehman JR, Webb DM, Tsareva TS, Haynes JS, Thacker BJ, Emerson SU: A novel virus in swine is closely related to the human hepatitis E virus. Proc Natl Acad Sci USA 1997, 94:9860-9865.

7. Purcell RH, Emerson SU: Hepatitis E: an emerging awareness of an old disease. J Hepatol 2008, 48:494-503.

8. Ohnishi S, Kang JH, Maekubo H, Arakawa T, Karino Y, Toyota J, Takahashi K, Mishiro S: Comparison of clinical features of acute hepatitis caused by hepatitis E virus (HEV) genotypes 3 and 4 in Sapporo, Japan. Hepatol Res 2006, 36:301-307.

9. Inoue J, Nishizawa T, Takahashi M, Aikawa T, Mizuo H, Suzuki K, Shimosegawa T, Okamoto H: Analysis of the full-length genome of genotype 4 hepatitis $E$ virus isolates from patients with fulminant or acute self-limited hepatitis E. J Med Virol 2006, 78:476-484.

10. Mizuo H, Yazaki Y, Sugawara K, Tsuda F, Takahashi M, Nishizawa T, Okamoto $\mathrm{H}$ : Possible risk factors for the transmission of hepatitis $\mathrm{E}$ virus and for the severe form of hepatitis E acquired locally in Hokkaido, Japan. J Med Virol 2005, 76:341-349.

11. Takahashi M, Nishizawa T, Yoshikawa A, Sato S, Isoda N, Ido K, Sugano K, Okamoto $\mathrm{H}$ : Identification of two distinct genotypes of hepatitis $\mathrm{E}$ virus in a Japanese patient with acute hepatitis who had not travelled abroad. I Gen Virol 2002, 83:1931-1940.

12. Zhu YM, Dong SJ, Si FS, Yu RS, Yu XM, Zou SX, Li Z: Swine and human hepatitis E virus (HEV) infection in China. J Clin Virol 2011, 52:155-157.

13. Si FS, Zhu YM, Dong SJ, Yu SS, Yu RS, Shen SY, Yang Q, Li Z: Full genomic sequence analysis of swine genotype 3 hepatitis $E$ virus isolated from Shanghai. Virus Res 2009, 144:290-293.

14. Zuker M: Mfold web server for nucleic acid folding and hybridization prediction. Nucleic Acids Res 2003, 31:3406-3415.

15. Huang YW, Opriessnig T, Halbur PG, Meng XJ: Initiation at the third inframe AUG codon of open reading frame 3 of the hepatitis $E$ virus is essential for viral infectivity in vivo. J Virol 2007, 81:3018-3026.

16. Fu H, Wang L, Zhu Y, Geng J, Li L, Wang X, Bu Q, Zhuang H: Analysing complete genome sequence of swine hepatitis $E$ virus (HEV), strain CHN-XJ-SW13 isolated from Xinjiang, China: putative host range, and disease severity determinants in HEV. Infect Genet Evol 2011, 11:618-623.

17. Ning H, Niu Z, Yu R, Zhang P, Dong S, Li Z: Identification of genotype 3 hepatitis $E$ virus in fecal samples from a pig farm located in a Shanghai suburb. Vet Microbiol 2007, 121:125-130. 
18. Wang Y: Epidemiology, molecular biology and zoonosis of genotype IV hepatitis E in China. Chin J Epidemiol 2003, 24:618-622.

19. Zhang A, Purcel RH, Emerson SU: Identification of the 5 ' terminal sequence of the SAR-55 and MEX-14 strains of hepatitis E virus and confirmation that the genome is capped. J Med Virol 2001, 65:293-295.

20. Purdy MTA, Huang C, Yarbough P, Reyes G: Hepatitis E virus: a non-enveloped member of the 'alpha- like' RNA virus supergroup. Sem Virol 1993, 4:319-326.

21. Fujiwara K, Yokosuka O, Ehata T, Saisho H, Saotome N, Suzuki K, Okita K, Kiyosawa K, Omata M: Association between severity of type A hepatitis and nucleotide variations in the 5 ' non-translated region of hepatitis A virus RNA: strains from fulminant hepatitis have fewer nucleotide substitutions. Gut 2002, 51: 82-88.

22. Agrawal S, Gupta D, Panda SK: The 3' end of hepatitis E virus (HEV) genome binds specifically to the viral RNA-dependent RNA polymerase (RdRp). Virology 2001, 282:87-101.

23. Graff J, Nguyen H, Kasorndorkbua C, Halbur PG, St Claire M, Purcell RH, Emerson SU: In vitro and in vivo mutational analysis of the 3 '-terminal regions of hepatitis E virus genomes and replicons. J Virol 2005, 79:1017-1026.

24. Koonin EV, Gorbalenya AE, Purdy MA, Rozanov MN, Reyes GR, Bradley DW: Computer-assisted assignment of functional domains in the nonstructural polyprotein of hepatitis $\mathrm{E}$ virus: delineation of an additional group of positive-strand RNA plant and animal viruses. Proc Natl Acad Sci USA 1992, 89:8259-8263.

25. Pudupakam RS, Huang YW, Opriessnig T, Halbur PG, Pierson FW, Meng XJ: Deletions of the hypervariable region (HVR) in open reading frame 1 of hepatitis $E$ virus do not abolish virus infectivity: evidence for attenuation of HVR deletion mutants in vivo. J Virol 2009, 83:384-395.

26. Zafrullah M, Ozdener MH, Kumar R, Panda SK, Jameel S: Mutational analysis of glycosylation, membrane translocation, and cell surface expression of the hepatitis E virus ORF2 protein. J Virol 1999, 73:4074-4082.

27. Tam AW, Smith MM, Guerra ME, Huang CC, Bradley DW, Fry KE, Reyes GR: Hepatitis E virus (HEV): molecular cloning and sequencing of the fulllength viral genome. Virology 1991, 185:120-131.

28. Córdoba L, Huang YW, Opriessnig T, Harral KK, Beach NM, Finkielstein CV, Emerson SU, Meng XJ: Three amino acid mutations (F51L, T59A, and S390L) in the capsid protein of the hepatitis E virus collectively contribute to virus attenuation. J Virol 2011, 85:5338-5349.

29. Guu TS, Liu Z, Ye Q, Mata DA, Li K, Yin C, Zhang J, Tao YJ: Structure of the hepatitis $E$ virus-like particle suggests mechanisms for virus assembly and receptor binding. Proc. Natl Acad Sci USA 2009, 106:12992-12997.

30. Yamashita T, Mori Y, Miyazaki N, Cheng RH, Yoshimura M, Unno H, Shima R, Moriishi K, Tsukihara T, Li TC, Takeda N, Miyamura T, Matsuura Y: Biological and immunological characteristics of hepatitis E virus-like particles based on the crystal structure. Proc Natl Acad Sci USA 2009, 106:12986-12991.

31. Pugachev KV, Galinski MS, Frey TK: Infectious CDNA clone of the RA27/3 vaccine strain of rubella virus. Virology 2000, 273:189-197.

32. Van Loon AA, de Haas N, Zeyda I, Mundt E: Alteration of amino acids in VP2 of very virulent infectious bursal disease virus results in tissue culture adaptation and attenuation in chickens. J Gen Virol 2002, 83:121-129.

33. Emerson SU, Nguyen HT, Torian U, Burke D, Engle R, Purcell RH: Release of genotype 1 hepatitis $E$ virus from cultured hepatoma and polarized intestinal cells depends on open reading frame 3 protein and requires an intact PXXP motif. J Virol 2010, 84:9059-9069.

34. Cao DJ, Huang YW, Meng XJ: The nucleotides on the stem-loop RNA structure in the junction region of the hepatitis $\mathrm{E}$ virus genome are critical for virus replication. J Virol 2010, 84:13040-13044.

35. Shukla P, Nguyen HT, Torian U, Engle RE, Faulk K, Dalton HR, Bendall RP, Keane FE, Purcell RH, Emerson SU: Cross-species infections of cultured cells by hepatitis $E$ virus and discovery of an infectious virus-host recombinant. Proc Natl Acad Sci USA 2011, 108:2438-2443.

36. Geng J, Fu H, Wang L, Bu Q, Liu P, Wang M, Sui Y, Wang X, Zhu Y, Zhuang $\mathrm{H}$ : Phylogenetic analysis of the full genome of rabbit hepatitis $E$ virus (rbHEV) and molecular biologic study on the possibility of cross species transmission of rbHEV. Infect Genet Evol 2011, 11:2020-2025.

doi:10.1186/1743-422X-9-264

Cite this article as: Zhu et al:: Determination of the full-genome sequence of hepatitis E virus (HEV) SAAS-FX17 and use as a reference to identify putative HEV genotype 4 virulence determinants. Virology Journal 2012 9:264.

\section{Submit your next manuscript to BioMed Central and take full advantage of:}

- Convenient online submission

- Thorough peer review

- No space constraints or color figure charges

- Immediate publication on acceptance

- Inclusion in PubMed, CAS, Scopus and Google Scholar

- Research which is freely available for redistribution 